\title{
A comparative study of sertraline dosages, plasma concentrations, efficacy and adverse reactions in Chinese versus Caucasian patients
}

\begin{abstract}
This prospective 6-week study examined the differences in dosage and steady state plasma concentrations of sertraline in Chinese versus Caucasian depressed patients. Two groups of Chinese patients from different geographical sites and a group of Caucasian patients were evaluated with clinical measures during an initial dose of $50 \mathrm{mg} /$ day, with subsequent doses adjusted clinically. The results of 17 Australian Chinese (ACHI), 13 Malaysian Chinese (MCHI) and 15 Australian Caucasians (AC) were analysed. Despite controlling for weight, the AC subjects received a significantly higher dose than both the ACHI $(\mathrm{P}=0.002)$ and the $\mathrm{MCHI}$ groups $(\mathrm{P}=0.012)$. However, the mean sertraline concentration to dose ratios at weeks 1 and 6 were not significantly different between the three groups. Sertraline was effective and well tolerated in both ethnic groups with few adverse events. Although there was a lack of difference between groups in the pharmacokinetic results, Chinese depressed patients appeared to require lower dosages with consequently lower plasma concentrations of sertraline compared to Caucasian patients to achieve clinical efficacy. Further studies of the dosages, kinetics and adverse effects of selective serotonin reuptake inhibitors linked with genotyping are necessary.
\end{abstract}

Keyword: Antidepressant response; Ethnicity; Pharmacogenetics; Sertraline 